\title{
3 Research Square

\section{An extended family of perforated submicrometer hollow or core-shell plasmonic particles as antireflective synthetic brochosomes}

\section{Zoran Jakšić ( $\sim$ jaksa@nanosys.ihtm.bg.ac.rs )}

Institute of Chemistry, Technology and Metallurgy, University of Belgrade, Serbia https://orcid.org/0000-0003-4242-8809

\section{Marko Obradov}

Institute of Chemistry, Technology and Metallurgy, University of Belgrade, Serbia https://orcid.org/0000-0001-8559-9048

\section{Olga Jakšić}

Institute of Chemistry, Technology and Metallurgy, University of Belgrade, Serbia https://orcid.org/0000-0002-0937-3677

\section{Dragan Tanasković}

Institute of Chemistry, Technology and Metallurgy, University of Belgrade, Serbia https://orcid.org/0000-0003-2606-219X

\section{Research Article}

Keywords: Biomimetics, Plasmonics, Antireflection, Brochosomes, Cicadellidae

Posted Date: October 15th, 2021

DOl: https://doi.org/10.21203/rs.3.rs-970931/v1

License: (c) (1) This work is licensed under a Creative Commons Attribution 4.0 International License. Read Full License 


\section{Abstract}

We investigated different biomimetic structures inspired by natural brochosome powders which appear on the bodies and wings of leafhoppers (insects from the Cicadellidae family). All structures we analyzed are roughly spherical, with diameters $200 \mathrm{~nm}$ to $1000 \mathrm{~nm}$, with a core and a shell made of different materials and the core perforated with subwavelength holes with diameters of the order of tens of nanometers. We extended the range of possible designs, geometries and materials of synthetic brochosomes, inspired by their natural counterparts found as powders secreted by various species of leafhoppers. We performed simulation of the optical properties of the structures using the finite element method. We found out that our approach ensures the design of highly efficient omnidirectional ultraantireflective diffractive powders with subwavelength apertures. The reflectivity of $600 \mathrm{~nm}$ diameter holey spheres does not exceed $0.02 \%$ in $500-600 \mathrm{~nm}$ range. We showed that planar arrays of plasmonic-based artificial brochosomes exhibit a rich optical behavior, including effective refractive index below unity and even below zero at longer wavelengths. Such metamaterial-like behavior contributes to the multifunctionality of our synthetic brochosomes which can already serve as antireflective, superhydrophobic and highly porous structures controllable by design. This kind of versatility shows potentials for numerous practical uses. A major part of the novel functionalities stems from the use of nanocomposites containing free-electron conductors (plasmonic materials). Thus we arrived at a toolbox for the design of highly customizable antireflective layers. Potential fields of use include photodetectors, photoelectrochemistry, photocatalysis and general microoptoelectromechanical (MOEMS) systems.

\section{Introduction}

Antireflective (AR) properties and cloaking are of crucial interest in many optical and photonic devices and systems. They are indispensable for the performance improvement of various photodetectors and find their application everywhere where it is necessary to increase the input light flux (Raut et al. 2011; Jakšić 2014).

There is a plethora of different approaches to decreasing reflection and increasing absorption. Historically, the oldest method is the use of interference ultrathin films that may be single- or multilayered (Macleod 2010). The next large group are various diffractive antireflective surface profiles. With the advent of plasmonics and metamaterials, new highly efficient structures appeared, mostly based on subwavelength diffractive elements utilizing metamaterials - the superabsorbers (Aydin et al. 2011). A typical metamaterial superabsorber has its top plasmonic surface structured - e.g. riddled with holes and has a bottom metallic layer, divided from the top by a dielectric spacer. Another family of synthetic antireflective structures includes the bioinspired ones (Han et al. 2016), the basic function of many of these overlapping in a certain degree with the diffractive antireflective surface profiles. One of the earliest examples that caught the attention of the scientific community were the so-called moth-eye structures periodic diffractive structures mimicking the antireflective relief found in the eyes of moths (Clapham and Hutley 1973). 
In 2017 a new approach to bioinspired antireflective structure has been proposed, based on plasmonics and metamaterials: the use of synthetic brochosomes as omnidirectional ultra-antireflective diffractive structures (Yang et al. 2017). It immediately attracted the attention of researchers and a number of papers appeared dealing with the various practical applications of synthetic brochosomes - e.g. (Ding et al. 2019; Lei et al. 2020; Hua et al. 2021; Li et al. 2021).

Natural brochosomes are submicrometer hollow spherical submicrometer particles usually consisting of proteins and lipids, their surfaces riddled with nanoscale apertures. They were first described as early as in 1950ties (Tulloch and Shapiro 1954) and were studied in depth by Rakitov and collaborators (Rakitov 1995; Rakitov 2000; Rakitov 2004; Rakitov and Gorb 2013). They are produced by some insects (e.g. leafhoppers - Hemiptera: Cicadellidae) and cover them as integumental powders. They simultaneously perform a role of superhydrophobic protection against sticking the insect to plants sap (Rakitov and Gorb 2013) and serve as antireflective coatings in the visible to reduce observability by predatory species (a natural cloaking device) (Yang et al. 2017).

A procedure for the microfabrication of synthetic brochosome-like structures was described by Yang et al (Yang et al. 2017). They deposited sacrificial nanospheres on the plasmonic shells of core-shell submicrometer particles at various depths and then etched them, leaving round holes in the surface. Other works dealing with the micro/nanofabrication of synthetic brochosomes include (Sukamanchi et al. 2017; Borja 2018; Ding et al. 2019; Lei et al. 2020; Shih et al. 2020; Li et al. 2021). Most of these make use of the fact that when fabricating synthetic brochosomes one is not limited by the materials appearing in the natural structures, which ensures a wider range of available properties and thus extended possibilities for practical utilization.

The experimental applications of synthetic brochosomes proposed so far show their true multifunctionality and include not only omnidirectional antireflective layers (Yang et al. 2017; Borja 2018; Lei et al. 2020; Shih et al. 2020; Li et al. 2021) but also superhydrophobic and superoleophilic particles and surfaces (Sukamanchi et al. 2017), electrochromic materials (Hua et al. 2021), omnidirectional surface-enhanced Raman scattering substrates (Ding et al. 2019), photoanodes for photoelectrochemical hydrogen production (Pan et al. 2019), etc. At that, this research field is only in its nascence, the first paper about the fabrication being published a few years ago (Yang et al. 2017). Thus it is reasonable to assume that new applications will continue to appear for at least some time. The variety of the practical implementations described so far confirms the multifunctionality of synthetic brochosomes and an obvious real-life interest for them.

In this work we first consider various alternative designs for synthetic brochosomes, both those found in nature and those only loosely inspired by the biological ones. We used free and open-source computer graphics environment Blender to design and render our structures. We modeled their electromagnetic properties like scattering parameters (transmission and reflection coefficients) of interest for their antireflective behavior using finite element method (FEM). We also extracted the effective optical parameters of the synthetic brochosomes from the simulated complex transmittance and reflectance. 


\section{Some Designs Of Synthetic Brochosomes}

We present here two groups of designs for synthetic brochosomes. One group are more or less replicas of the natural structures secreted as integumental protective submicrometer particles by leafhoppers. Another large group are the synthetic brochosomes that do not exhibit a direct correspondence with the natural ones. Instead of it, they share a number of general properties, being hollow or dielectric-filled (core-shell) sphere-like shells with subwavelength apertures on their surfaces. Among examples of such alternative designs are the structures designed, modeled and fabricated by (Yang et al. 2017). We may say for the members of the latter group that they draw the basic inspiration from nature, but the exact geometries and materials will vary wildly and the departures from the natural structures may be quite significant. In other words, these synthetic structures are inspired by their natural counterparts, some more loosely than the others.

For the both groups we generated appropriate 3D models in the free and open-source software package for computer graphics Blender ver. 2.82. Some chosen examples of the investigated structures are further described.

Figure 1 represents a simplified synthetic analogue of the natural brochosomes secreted by e.g. the species Paraulacizes irrorata and Oncometopia orbona (Rakitov 2004). Similar shapes are actually ubiquitous among leafhoppers and are encountered in a majority of the integumental powders of various Cicadellidae. Their dimensions in nature (diameter) may vary between $200 \mathrm{~nm}$ and $600 \mathrm{~nm}$, but some larger specimens even reach up to $5 \mathrm{~mm}$. The left part of Fig. 1 shows the templating that would be necessary to synthesize the shapes as shown in Fig. 1 right. The white sphere represents the core which is either filled with dielectric or hollow, the pink shapes with trapeze-like cross-section are actually truncated hexagonal pyramids that serve as sacrificial structures, while light blue part represents a conductive plasmonic layer deposited after the sacrificial structures. The final synthetic brochosomes have their extruded parts (plasmonic material "walls") similar to a honeycomb, while its layout is icosahedral, not unlike the fullerene (buckyball) shape. Obviously, such templated structures with nanometer-scale details would be extremely difficult to fabricate, albeit their effectiveness is already proven in nature. Thus instead of these shapes, we consider much simpler geometries, easier to produce using conventional microfabrication and nanofabrication by sacrificial nanoscale spherules in a manner akin to that presented in (Yang et al. 2017).

Figure 2 shows a relatively simple synthetic brochosome structure that can be obtained in a manner quite similar to that described by Yang et al (Yang et al. 2017).

As in (Yang et al. 2017), templating can be done using nanoscale sacrificial spherules densely packed on the surface of a larger sphere. The final shape after etching has a cratered appearance, its hemispherical pits being scattered across the plasmonic core-shell surface in a rather random manner. Very similar shapes are found in nature in large brochosomes used for egg powdering in Oncometopia genus leafhoppers, an average diameter of a powder particle being about $1 \mathrm{~mm}$. 
We further consider some synthetic brochosomes which actually represent variations of the one shown in Fig. 2. Several possible forms are shown in Fig. 3. Basically, each of the particles is a submicrometersized plasmonic shell with holes of varying diameters templated by sacrificial spherules of different sizes and placed at different depths.

The number of possible combinations is literally endless, even when one considers holey core-shell geometry variations only. Even natural structures are encountered in a lot of different forms and sizes, a majority of which is not even mentioned here. A review of some of numerous shapes and kinds of brochosomes can be found in (Rakitov 2004). In addition to that vast diversity, novel properties are also obtained by choosing building materials outside the nature toolbox, like in our case (as far as the authors know, there are no known plasmonic biological structures).

\section{Results And Discussion}

We simulated the optical properties of our proposed synthetic brochosomes by FEM using the Comsol Multiphysics software package - RF Module. We calculated the spectral dispersion of scattering parameters (coefficients of transmission and reflection) and the spatial distribution of the electromagnetic field intensity. The holey plasmonic shell was illuminated from the top (the case of normal incidence of the light beam). We assumed that the plasmonic material was gold. In order to obtain more realistic results, its optical parameters were modeled using experimental data from literature (Rakić et al. 1998) rather than using the idealized Drude model that deviates in a certain degree from the reality. We assumed that the dielectric core within the particles was air (i.e. refractive index $n=1$ ).

We first considered the scattering parameters of the shapes shown in Fig. 3 top. In order to check if the antireflective properties appear even for the simplest cases, we performed our simulations for a shell with 6 holes, an opposing pair on the $x, y$ and $z$ axis each. The shell diameter was $600 \mathrm{~nm}$, the wall thickness was $20 \mathrm{~nm}$ and the hole diameter was $160 \mathrm{~nm}$. We considered a 2D square array of holey shells, the distance between two neighboring shells being $200 \mathrm{~nm}$. The transmission and reflection coefficient are shown in Fig. 4. In Fig. 4 left one can see that there are two bands with very low reflection coefficients, at 500-600 nm (green-yellow light) and above $1100 \mathrm{~nm}$ (near infrared). Fig. 4 right is an extract from Fig. 4 left, shown in the $500-700 \mathrm{~nm}$ range and in logarithmic scale to stress the low values of the reflection coefficient at the lowest wavelengths (even reaching below $0.02 \%$ between $500 \mathrm{~nm}$ and $600 \mathrm{~nm}$ ). The reflection drop in near infrared (the rightmost part of Fig. 4 left) is due to the plasmonic particles starting to behave as an effective medium mostly consisting of dielectric (air) - the well-known artificial dielectric effect (Brown 1953; Mendis et al. 2016) - thus decreasing their absorption with wavelength and becoming transparent. Since this phenomenon does not contribute to increased absorption, it is of no immediate interest for us here.

The spatial distribution of the electric field is shown in Fig. 5 for a square array of perforated hollow particles and on the surface of a single particle from that array. Two operating wavelengths of interest were considered, $550 \mathrm{~nm}$ and $680 \mathrm{~nm}$. 
The propagating waves are coupled to the golden spheres through nanoaperture scattering, similar to that first described by Ebessen and coworkers in their seminal paper on extraordinary transmission hole arrays (Ebessen et al. 1998). The difference is that in our case, due to the specific geometry of the structures, the increase of the wavevector of the incident waves results in generation of localized surface plasmons polaritons (SPP) on the plasmonic shells. This effect can readily be observed in Fig. 5 at 550 $\mathrm{nm}$ (bottom) where a distinct dipole radiative pattern is observed around the nanoaperture. Inside the sphere scattered field couples with quadrupole resonant mode of the shell which in turn couples with adjacent particles resulting in leaky modes (weak confinement). On the other hand, at $680 \mathrm{~nm}$ the shell material (gold) has a much higher refractive index, resulting in much more confined spatial field distributions. Nanoaperture scattering results in highly localized "pockets" of EM radiations due to increased field penetration into the structure. At the end of the process the localized SPP are absorbed in the highly absorptive metal of the shell both from outside and from within the sphere. The result is a large drop of the reflection coefficient in the $500-600 \mathrm{~nm}$ range as seen in Fig. 4.

We further performed extraction of the effective optical parameters for our planar arrays of holey spheres. We calculated the complex values of the effective relative dielectric permittivity $(\varepsilon)$, effective relative magnetic permeability $(\mu)$ and effective refractive index $(n)$. To this purpose we used the approach of Smith and coworkers (Smith et al. 2002) and applied it to our simulated complex transmittance and reflectance. Fig. 6 shows the extracted effective parameters.

The extraordinary spectral behavior of the extracted optical parameters of the planar array of synthetic brochosomes is readily noticed in Fig. 6. Remarkably, their values mostly remain below unity. In the 500$600 \mathrm{~nm}$ range both the real and the imaginary part of the effective refractive index remain relatively close to zero, never exceeding 0.1 , but are always positive. However, in the range above $1 \mu \mathrm{m}$ the real part of the effective refractive index drops below zero, meaning that the array behaves as a double negative metamaterial. Such rich behavior of the spectral dispersion points out to numerous potential practical applications of plasmonic synthetic brochosomes.

\section{Conclusion}

In this work we proposed some extensions and generalizations to bio-inspired geometries of synthetic brochosomes containing plasmonic holey shell. We simulated the electromagnetic parameters of the structures using the FEM approach. We succeeded in obtaining very low reflection coefficients in a wavelength range of interest (especially yellow and green part of the spectrum). We showed that even in a very simple case of just 6 subwavelength holes the planar arrays of plasmonic-based artificial brochosomes show a rich optical behavior, including effective refractive index below unity and even below zero at longer wavelengths. Such metamaterial-like behavior, together with their multifunctionality (they can serve as antireflective, superhydrophobic and highly porous structures with parameters controllable by design) points out to a versatility that may prove itself convenient for numerous microoptoelectromechanical (MOEMS) systems. In our further work we intend to extend our investigations to other geometries and plasmonic/dielectric material pairs. 


\section{Declarations}

\section{Conflict of interests}

The authors have no conflicts of interest to declare.

\section{Funding}

This work was supported by the Ministry of Education, Science and Technological Development of Republic of Serbia, Grant No: 451-03-9/2021-14/200026.

\section{References}

Aydin, K., Ferry, V. E., Briggs, R. M., Atwater, H. A.: Broadband polarization-independent resonant light absorption using ultrathin plasmonic super absorbers. Nature comm., 2(1), 1-7 (2011) https://doi.org/10.1038/ncomms1528

Borja, L. Synthesis replicates camouflaging-brochosome particle structure. MRS Bull., 43(1), 10 (2018) https://doi.org/10.1557/mrs.2017.308

Brown, J.: Artificial dielectrics having refractive indices less than unity. Proc. IEE-Pt IV, 100(5), 51-62 (1953) https://doi.org/10.1049/pi-4.1953.0009

Clapham, P. B., Hutley, M. C. Reduction of lens reflexion by moth eye principle. Nature 244, 281-282 (1973) https://doi.org/10.1038/244281a0

Ding, Q., Kang, Y., Li, W., Sun, G., Liu, H., Li, M., Ye, Z., Zhou, M., Zhou, J., Yang, S.: Bioinspired brochosomes as broadband and omnidirectional surface-enhanced Raman scattering substrates. J. Phys. Chem. Lett., 10(21), 6484-6491 (2019) https://doi.org/10.1021/acs.jpclett.9b02380

Ebbesen, T. W., Lezec, H. J., Ghaemi, H. F., Thio, T., Wolff, P. A.: Extraordinary optical transmission through sub-wavelength hole arrays. Nature, 391(6668), 667-669 (1998) https://doi.org/10.1038/35570

Han, Z. W., Wang, Z., Feng, X. M., Li, B., Mu, Z. Z., Zhang, J. Q., Niu, S. C., Ren, L. Q.: Antireflective surface inspired from biology: a review. Biosurf. Biotribol., 2(4), 137-150 (2016)

https://doi.org/10.1016/j.bsbt.2016.11.002

Hua, C., Cheng, Z., Ma, Y., He, H., Xu, G., Liu, Y., Yang, S., Han, G.: Enhanced Electrochromic Tungsten Oxide by Bio-Inspired Brochosomes. J. Electrochem. Soc., 168(4), 042503 (2021) https://doi.org/10.1149/1945$7111 / a b f 261$ 
Jakšić, Z.: Micro and Nanophotonics for Semiconductor Infrared Detectors. Springer: Switzerland (2014) https://doi.org/10.1007/978-3-319-09674-2

Lei, C. W., Chen, R. Y., Yang, H.: Leafhopper Wing-Inspired Broadband Omnidirectional Antireflective Embroidered Ball-Like Structure Arrays Using a Nonlithography-Based Methodology. Langmuir, 36(19), 5296-5302 (2020)

https://doi.org/10.1021/acs.langmuir.0c00634

Li, P. C., Chen, H. Y., Chiang, K. T., Yang, H.: Reversible embroidered ball-like antireflective structure arrays inspired by leafhopper wings. J. Colloid Interf. Sci., 599, 119-129 (2021) https://doi.org/10.1016/j.jcis.2021.04.079

Macleod, H. A.: Thin-film optical filters, 4th ed. CRC press, Boca Raton (2010) https://doi.org/10.1201/9781420073034

Mendis, R., Nagai, M., Wang, Y., Karl, N., Mittleman, D. M.: Terahertz artificial dielectric lens. Scientific reports, 6(1), 1-8 (2016) https://doi.org/10.1038/srep23023

Pan, Q., Zhang, H., Yang, Y., Cheng, C.: 3D Brochosomes-Like $\mathrm{TiO}_{2} / \mathrm{WO}_{3} / \mathrm{BiVO}_{4}$ Arrays as Photoanode for Photoelectrochemical Hydrogen Production. Small, 15(28), 1900924 (2019)

https://doi.org/10.1002/smll.201900924

Rakić, A. D., Djurišić, A. B., Elazar, J. M., Majewski, M. L.: Optical properties of metallic films for verticalcavity optoelectronic devices. Appl. Opt., 37(22) 5271-5283 (1998)

https://doi.org/10.1364/AO.37.005271

Rakitov, R. A.: The covering formed by brochosomes on the cuticle of leafhoppers (Homoptera, Cicadellidae). Entomol. Rev., 74(9), 90-103 (1995)

Rakitov, R. A.: Powdering of egg nests with brochosomes and related sexual dimorphism in leafhoppers (Hemiptera: Cicadellidae). Zool. J. Linnean Soc., 140(3), 353-381 (2004) https://doi.org/10.1111/j.10963642.2003.00103.x

Rakitov, R. A.: Secretion of brochosomes during the ontogenesis of a leafhopper, Oncometopia orbona (F.) (Insecta, Homoptera, Cicadellidae). Tissue and Cell, 32(1), 28-39 (2000) https://doi.org/10.1054/tice.1999.0084

Rakitov, R. A., Gorb, S. N.: Brochosomes protect leafhoppers (Insecta, Hemiptera, Cicadellidae) from sticky exudates. J. Roy. Soc. Interf., 10(87), 20130445 (2013) https://doi.org/10.1098/rsif.2013.0445

Raut, H. K., Ganesh, V. A., Nair, A. S., Ramakrishna, S.: Anti-reflective coatings: A critical, in-depth review. Energy \& Environm. Sci., 4(10), 3779-3804 (2011) https://doi.org/10.1039/C1EE01297E 
Shih, M.-S., Chen, H.-Y., Li, P.-C., Yang, H.: Broadband omnidirectional antireflection coatings inspired by embroidered ball-like structures on leafhoppers. Appl. Surf. Sci., 532, 147397 (2020). https://doi.org/10.1016/j.apsusc.2020.147397

Smith, D. R., Schultz, S., Markoš, P., Soukoulis, C. M.: Determination of effective permittivity and permeability of metamaterials from reflection and transmission coefficients. Physical review B, 65(19), 195104 (2002) https://doi.org/10.1103/PhysRevB.65.195104

Sukamanchi, R., Mathew, D., Kumar KS., S.: Durable superhydrophobic particles mimicking leafhopper surface: superoleophilicity and very low surface energy. ACS Sust. Chem. \& Eng., 5(1), 252-260 (2017) https://doi.org/10.1021/acssuschemeng.6b01413

Tulloch, G. S., Shapiro, J. E.: Brochosomes and leafhoppers. Science, 120 (3110), 232-232 (1954) https://doi.org/10.1126/science.120.3110.232

Yang, S., Sun, N., Stogin, B. B., Wang, J., Huang, Y., Wong, T. S.: Ultra-antireflective synthetic brochosomes. Nat. comm., 8(1), 1-8 (2017) https://doi.org/10.1038/s41467-017-01404-8

\section{Figures}
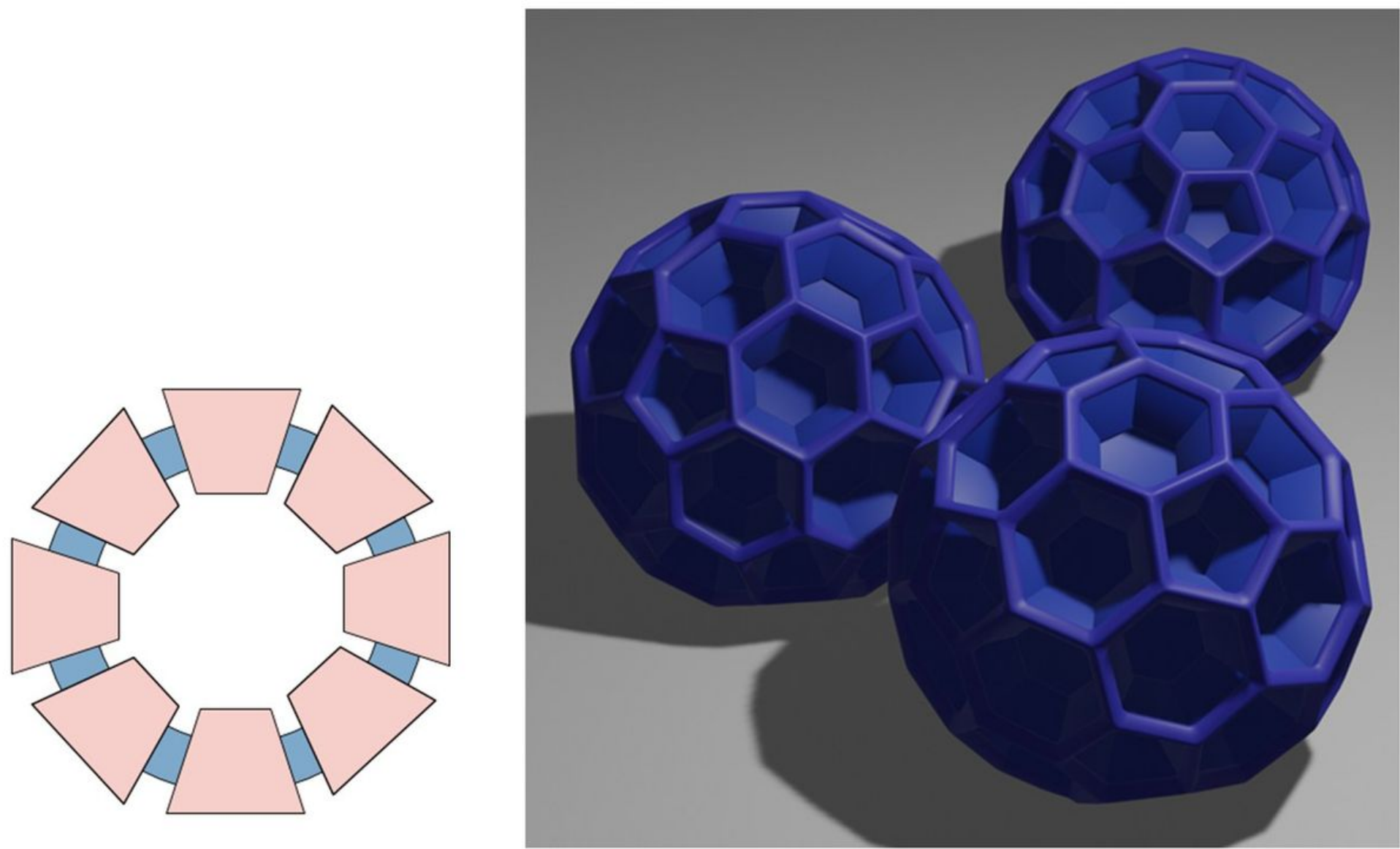

Figure 1 
Biomimetic core-shell submicrometer powder miming the brochosomes of the leafhopper tribe Proconiini and generally Cicadellidae family. Left: schematics of templated synthesis of the biomimetic core-shells. Blue annulus: plasmonic shell. Pale pink trapezes: sacrificial structures to be etched, leaving the holey shell. White: core, either hollow or filled with dielectric. Right: 3D CG render of biomimetic core-shells.
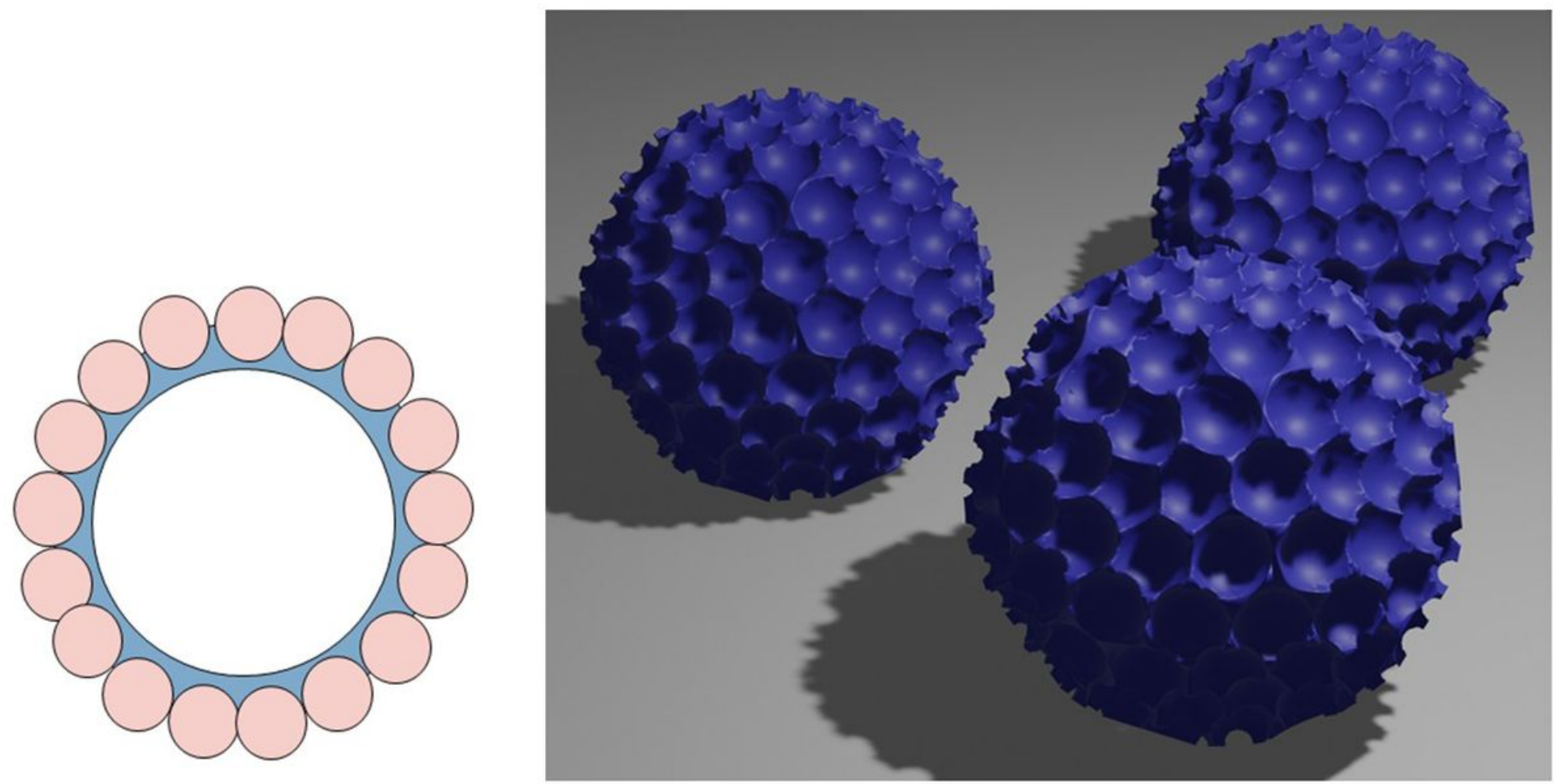

Figure 2

Biomimetic core-shell microscale powder miming the brochosomes of the leafhopper genus Oncometopia. Left: schematics of templated synthesis of the biomimetic core-shells. Blue annulus: plasmonic shell. Pale pink spheres: sacrificial structures to be etched, leaving the holey shell. White: core, either hollow or filled with dielectric. Right: 3D CG render of biomimetic core-shells. 

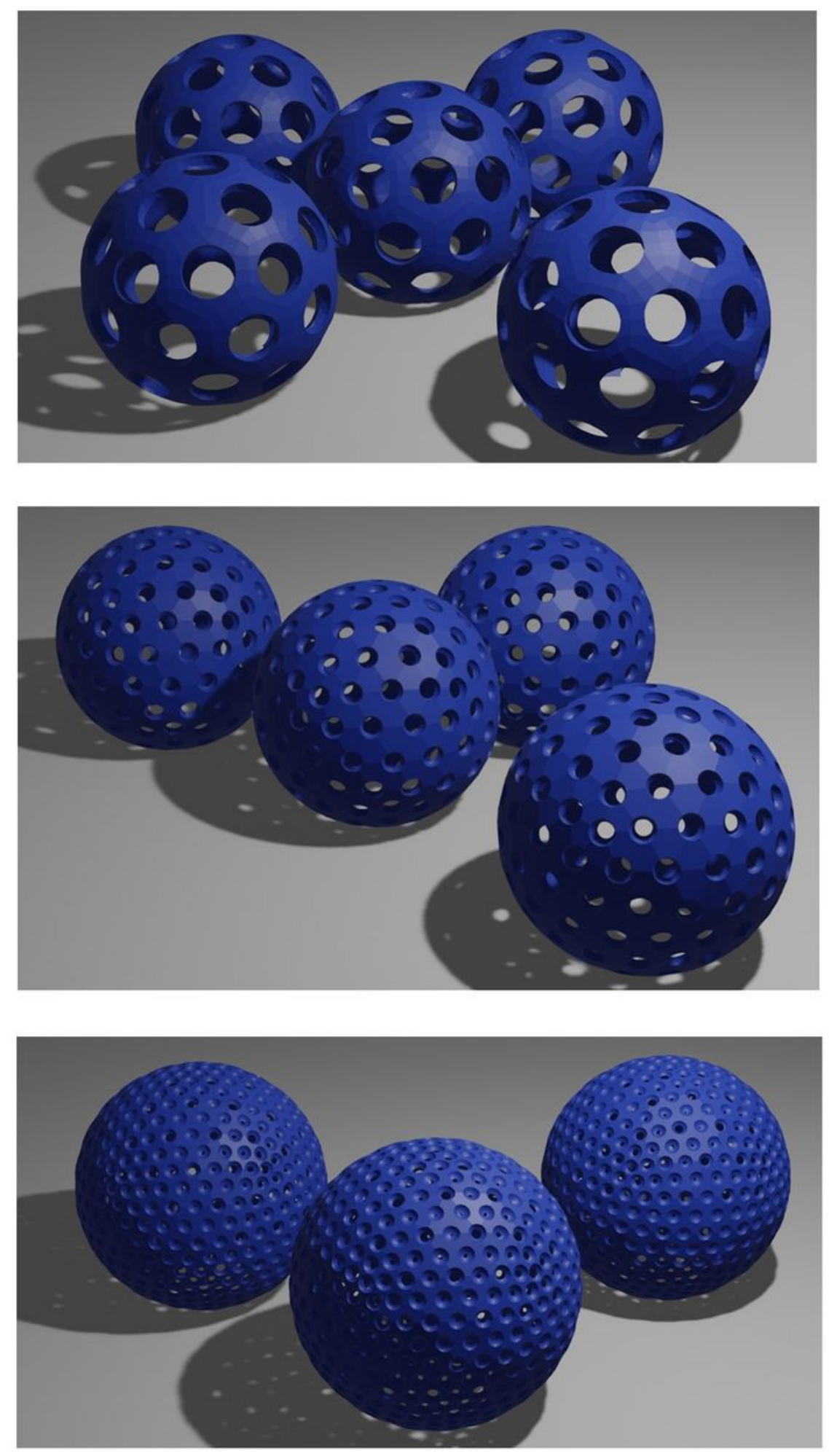

\section{Figure 3}

3D CG renders of biomimetic core-shell submicrometer powders consisting of hollow metal cores riddled with nanoscale apertures. Templating is done in a manner identical to that in Fig. 2, but with various sizes of spherules at different depths. Top: largest holes, middle: mid-sized holes. Bottom: smallest holes. 

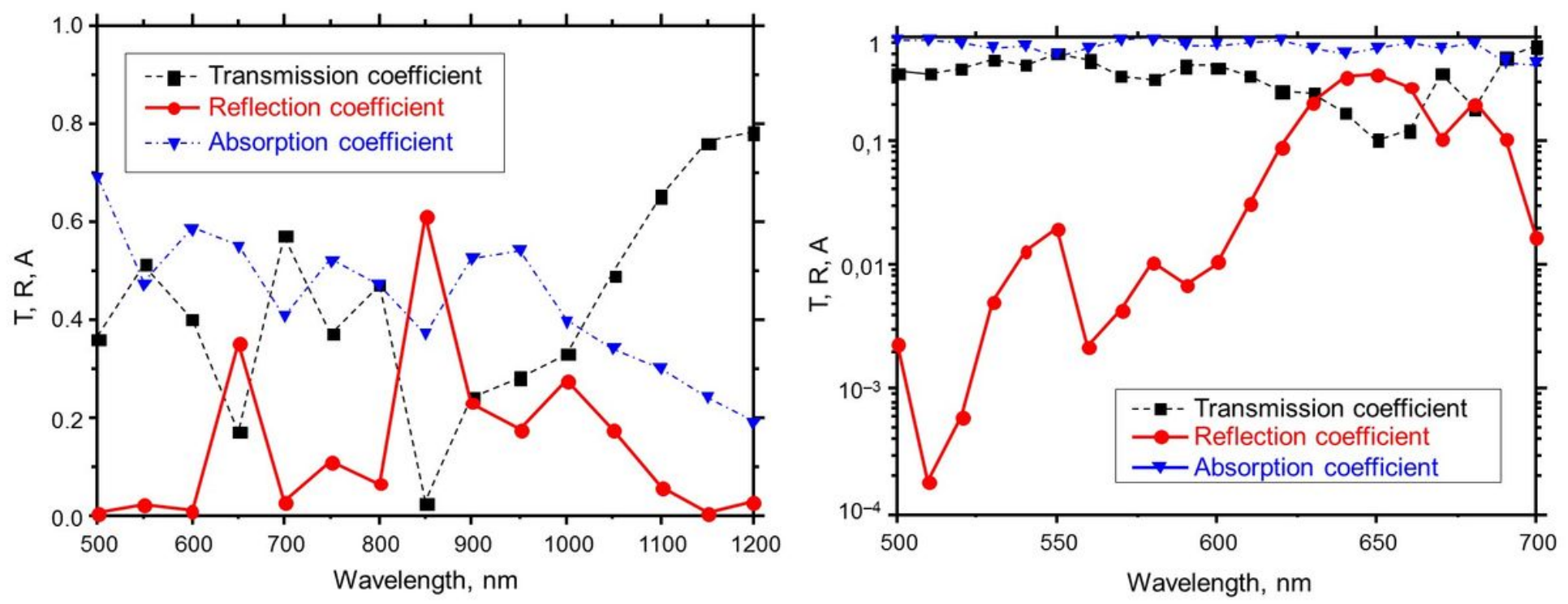

Figure 4

Spectral dependence of scattering parameters of synthetic brochosomes arranged in a square array of holey golden shells (shell diameter $600 \mathrm{~nm}$, wall thickness $20 \mathrm{~nm}$, hole diameter $160 \mathrm{~nm}, 6$ holes in total). Left: spectral reflection, transmission and absorption coefficients, linear scale. Right: Finer view of the same dependence as shown left, 500-700 nm range, logarithmic scale. 


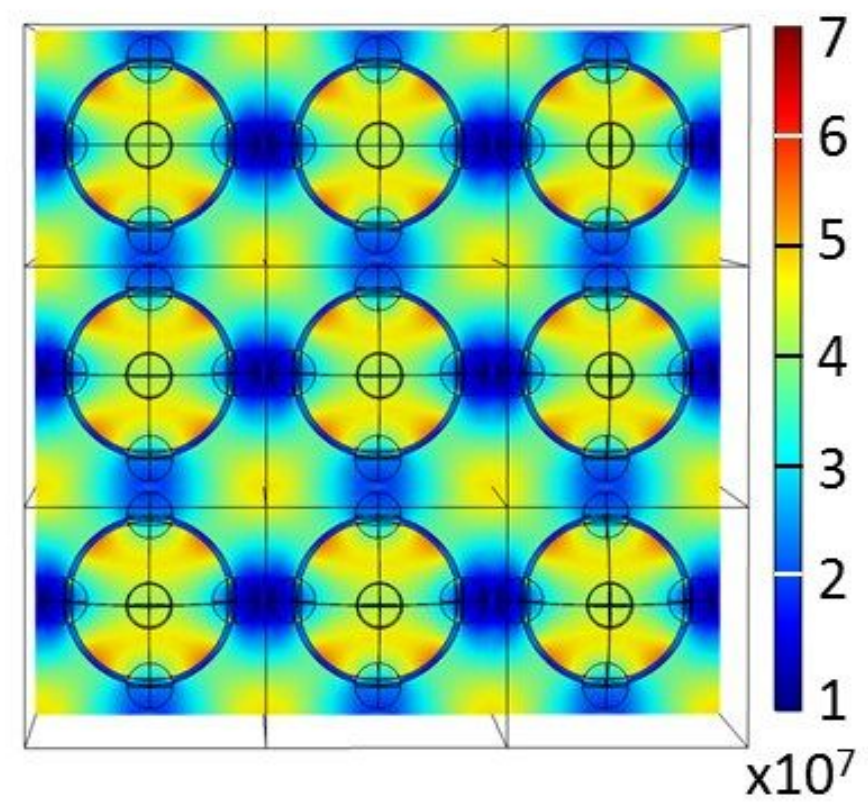

$550 \mathrm{~nm}$

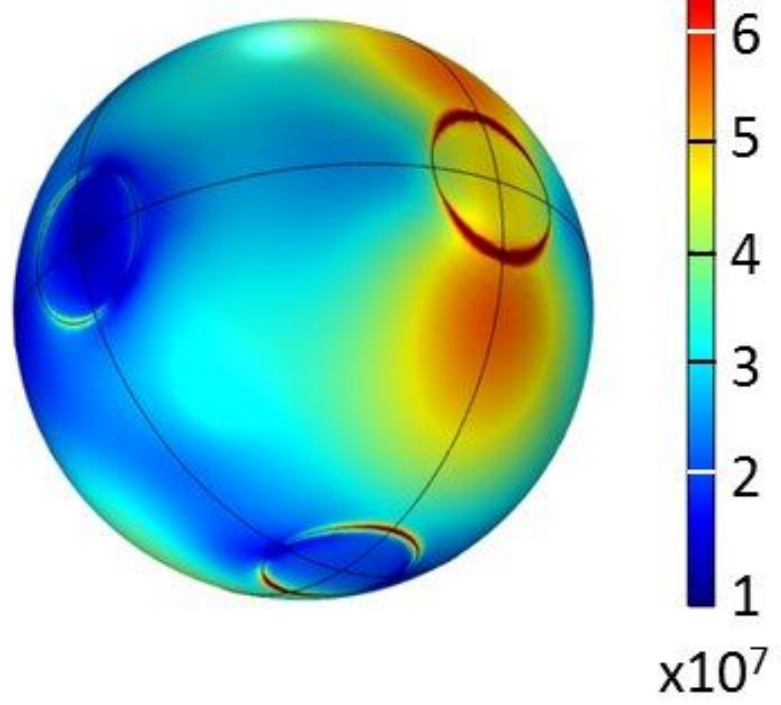

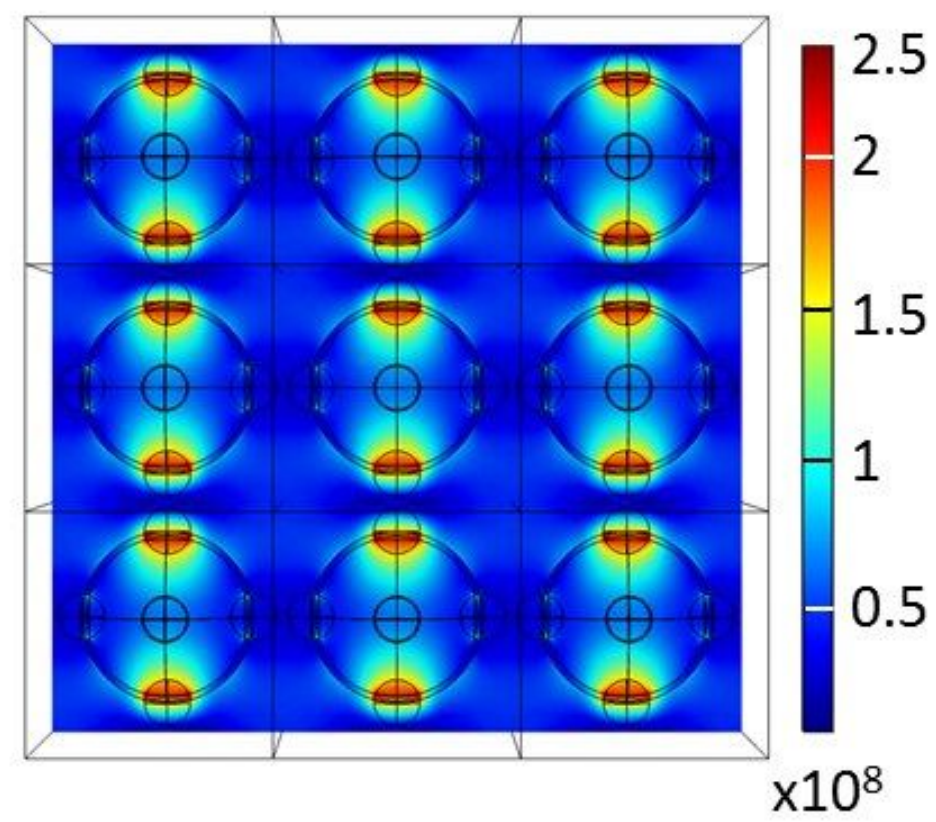

$680 \mathrm{~nm}$
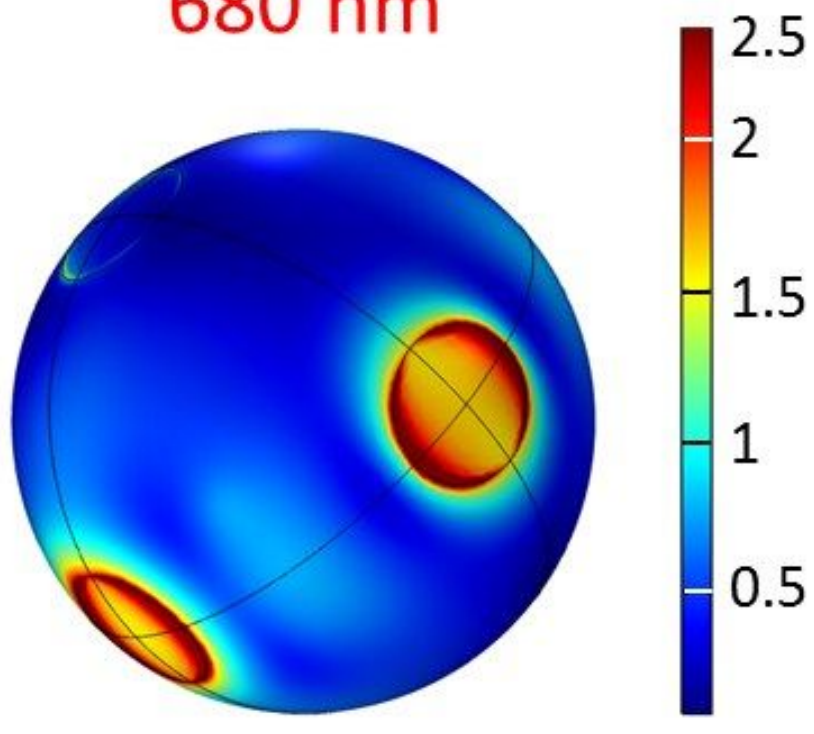

$x 10^{8}$

Figure 5

Spatial distribution of electric field in synthetic brochosomes. Top row: square array of holey shells. Bottom row: surface field of a singled out holey shell from the array above. Left column: operating wavelength $550 \mathrm{~nm}$. Right column: $680 \mathrm{~nm}$. 

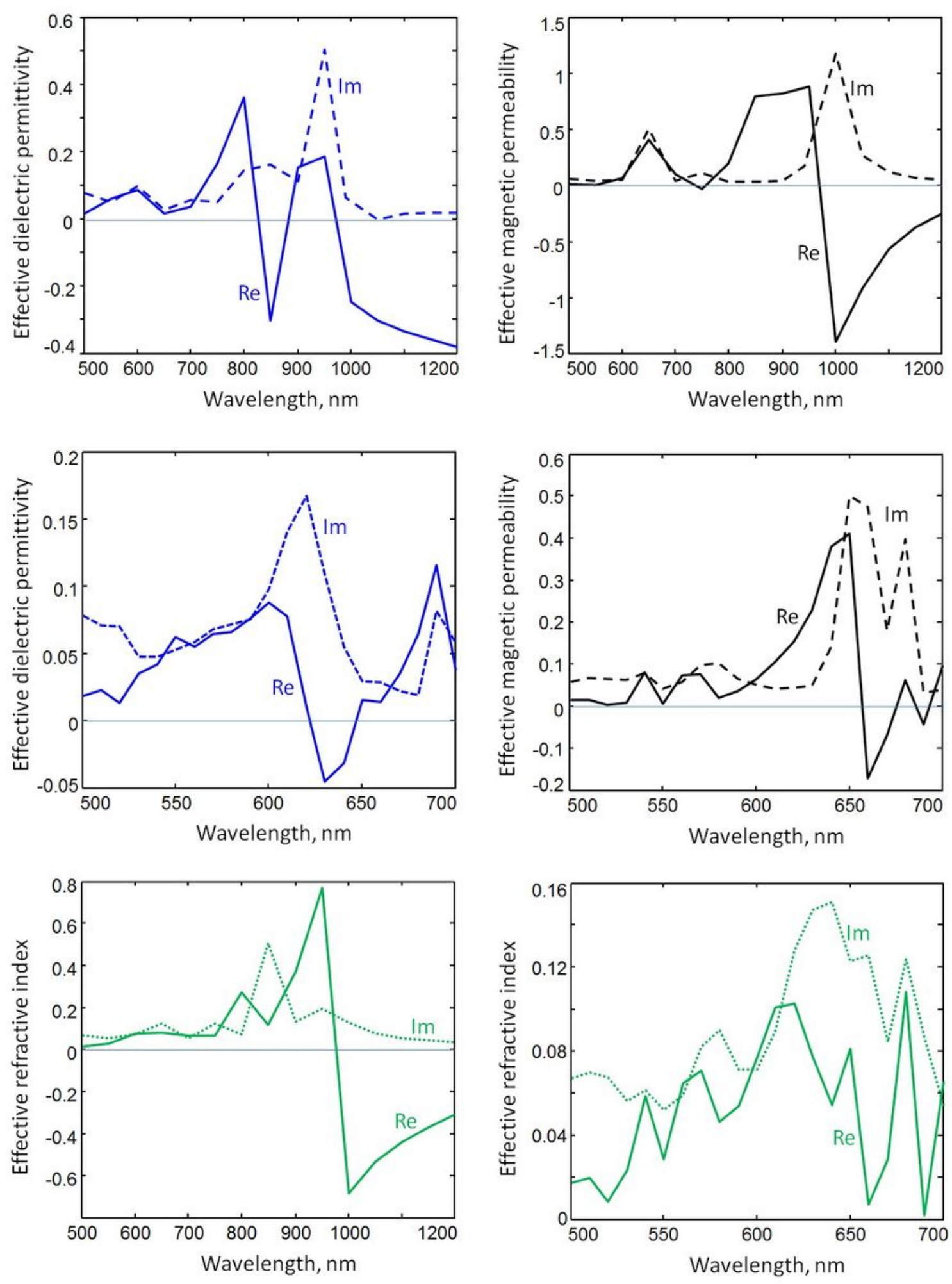

\section{Figure 6}

Extracted complex effective optical parameters of the planar array of holey plasmonic shells of synthetic brochosomes. Top row: effective epsilon and mu for the 500-1200 nm range. Middle row: effective epsilon and mu for the $500-700 \mathrm{~nm}$ range. Bottom row: effective refractive index for the $500-1200 \mathrm{~nm}$ range (left) and for the $500-700 \mathrm{~nm}$ range (right). 